\title{
GRUPOS FUNCIONALES EN LA DINÁMICA DEL BANCO DE SEMILLAS EN UN BOSQUE TEMPLADO DE LA CIUDAD DE MÉXICO, MÉXICO
}

\section{FUNCTIONAL GROUPS IN THE DYNAMICS OF THE SEED BANK IN A TEMPERATE FOREST OF MeXICo City, MeXICO}

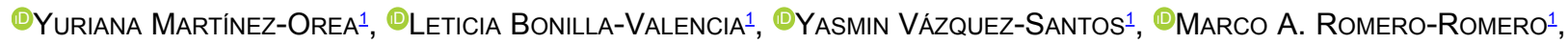

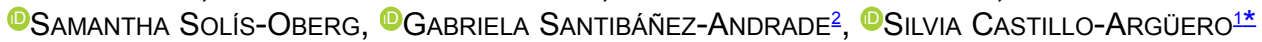

'Departamento de Ecología y Recursos Naturales, Facultad de Ciencias, Universidad Nacional Autónoma de México, México. ${ }^{2}$ Instituto Nacional de Investigaciones Forestales, Agrícolas y Pecuarias. México.

*Autor para correspondencia: silcas@ciencias.unam.mx

\section{Resumen}

Antecedentes: Este trabajo analiza el banco de semillas en diferentes sustratos (hojarasca y suelo) para conocer el efecto de la temporalidad y la orientación de la ladera sobre la abundancia y composición del banco y de la vegetación establecida en un bosque templado en la Ciudad de México.

Preguntas: ¿Qué factores abióticos determinan la germinación de nuevos individuos de distintos grupos funcionales?, ¿De qué manera esta información contribuye a la planeación de programas de conservación y restauración?

Descripción de datos: Este estudio se realizó en 30 parcelas establecidas en el bosque (ladera norte y sur) durante las dos temporadas del año. Sitio de estudio: Bosque templado de Quercus rugosa (L.) en la cuenca del río Magdalena, Cd. de México.

Métodos: Mediante una clasificación TWINSPAN se determinaron nueve grupos funcionales de banco de semillas y vegetación establecida. Mediante un análisis de Permutación de Respuesta Múltiple (MRPP) y un Análisis de Correspondencia Canónica (CCA) se determinó el efecto temporal y espacialmente sobre la abundancia y riqueza de los grupos funcionales.

Resultados: Se encontró una alta similitud dentro de cada grupo funcional y una alta disimilitud entre ellos. El Análisis de Varianza de Permutación Multivariado (PERMANOVA) mostró un efecto significativo de la temporada y la orientación sobre la identificación de los grupos funcionales.

Conclusiones: En términos de conservación la presencia de especies características y nativas implica que hay una alta resiliencia, sin embargo, la presencia de especies introducidas indica cambios en la composición del sistema, lo cual compromete el potencial de la regeneración natural.

Palabras clave: Germinación, hojarasca, reclutamiento, sustratos, temporalidad, vegetación establecida.

\begin{abstract}
Background: This research analyzes the seed bank of different substrates (litter and soil) and the standing vegetation to know the effects of the season and slope orientation on their abundance and composition in a temperate forest in Mexico City.

Questions and / or Hypotheses: Which abiotic factors determine the germination of new individuals of different functional groups? how this information can contribute in the planning of conservation and restoration programs? Which environmental factors determine germination and the recruiting of individuals? And how this knowledge could be considered in conservation programs?

Studied species / data description: This research was carried out in 30 plots established in the forest (North and South slopes) during both year seasons.

Study site and dates: Temperate forest of Quercus rugosa (L.) in the Magdalena river Basin, Mexico City.

Methods: Through a TWINSPAN classification nine functional groups were determined in the seed bank and the standing vegetation. Through a Multiple Response Permutation Analysis (MRPP) and a Canonical Correspondence Analysis (CCA) the environmental effect was determined, seasonally and spatially on the abundance and richness of the functional groups.

Results: A high similarity inside each functional group and a high dissimilarity between them were found. The Multivariate Permutation Variance Analysis (PERMANOVA) showed a significant effect of the season and of the slope orientation on the identification of the functional groups.

Conclusions: In terms of conservation the presence of characteristic and native species implies a high resilience, however the presence of introduced species indicates changes in the system composition, which jeopardizes the natural regeneration potential.

Key words: Established vegetation, germination, litter, recruiting, substrates, temporality.
\end{abstract}


Los bosques templados son uno de los ecosistemas con mayor deterioro por actividades antrópicas en todo el mundo (Hannah et al. 1995, Millar \& Stephenson 2005). En México son el segundo ecosistema más dominante $\mathrm{y}$ transformado (Sánchez-Colón et al. 2009). Los bosques cercanos a grandes zonas urbanas son los más sometidos a fuertes presiones y disturbios, como el cambio de uso de suelo (McKinney 2002). De manera que, la resiliencia de estos ecosistemas juega un papel importante en el mantenimiento de su funcionalidad.

La capacidad de regeneración de una comunidad, a partir del banco de semillas, disminuye drásticamente con el incremento de las actividades antrópicas, que en conjunto ocasionan la pérdida del hábitat. Esto favorece la colonización de diferentes tipos de especies, entre las que se encuentran las malezas nativas o introducidas, $y$ en consecuencia se generan cambios en la composición, abundancia y estructura de las comunidades (McKinney 2002, Tovar-Sánchez et al. 2004). Además, tiene implicaciones sobre las características del suelo, al modificar la estructura del mantillo, la cantidad de nutrientes, su grado de compactación, y capacidad de infiltración del agua de lluvia que en conjunto afectan negativamente la regeneración natural a partir del banco de semillas (Aide et al. 2000).

La dinámica y composición del banco de semillas de una comunidad vegetal determinan el potencial de la regeneración natural e influyen en la permanencia de las especies, así como en la trayectoria y destino de la sucesión secundaria (Martínez-Orea et al. 2013). La regeneración natural a partir de semillas depende de muchos factores, entre ellos los mecanismos de dispersión, la depredación, la formación de bancos de semillas, la latencia de las semillas y sus requerimientos particulares para germinar, así como también de los factores abióticos y de las estrategias biológicas que permiten el reclutamiento y establecimiento de nuevos individuos (Catovsky \& Bazzaz 2002, Catovsky et al. 2002). Para la regeneración de cualquier comunidad vegetal se requiere de un suministro constante de semillas, mismo que determina la transición entre la incorporación de semillas y su germinación (Primack \& Kang 1989). El banco de semillas es un reservorio evolutivo que depende de la historia de vida de las especies, la estructura de la vegetación, los disturbios naturales y antrópicos, la orientación de la ladera y otros procesos ecológicos y estocásticos; mediante los cuales se regula el flujo de semillas en el suelo (Martínez-Orea et al. 2013, ZepedaGómez et al. 2015). La dinámica y estructura de una comunidad depende de factores bióticos y abióticos, los cuales pueden actuar como filtros, limitando o facilitando la germinación y el establecimiento de las especies en un ecosistema (Catovsky et al. 2002).
Se ha comprobado que, en sitios con mayor recurrencia de disturbios, y como consecuencia cambios en las condiciones ambientales, la similitud tiende a aumentar entre el banco de semillas y la vegetación establecida, lo que refleja una mayor abundancia de especies características de la vegetación secundaria recién incorporadas al banco de semillas (Matus et al. 2003, Pakeman \& Small 2005). La mayoría de los estudios que analizan la relación entre el banco de semillas y la vegetación establecida han encontrado una discrepancia entre la composición de especies (Martínez-Orea et al. $\underline{2013})$.

En el banco de semillas se encuentran diferentes grupos funcionales de especies que se pueden establecer en ambientes particulares y bajo diferentes factores generando el complejo ensamblaje de las comunidades vegetales (Castellanos-Castro \& Bonilla 2011). Definiendo a los grupos funcionales como el conjunto de especies con atributos biológicos y estrategias de regeneración similares, que favorecen su éxito competitivo y tolerancia al estrés en condiciones ambientales similares (Dey et al. 2019). Por ejemplo, es el caso de especies catalogadas como malezas, o denominadas especies características que dependiendo del tipo de sustrato y perturbación pueden variar en sus abundancias, como respuesta a cambios en los factores abióticos (Verheyen et al. 2003).

Los bosques templados de Quercus spp., se caracterizan por una continua producción de hojarasca altamente lignificada, proveniente principalmente de árboles (Molofsky \& Augspurger 1992, Kostel-Hughes et al. 2005), esta hojarasca se descompone muy lentamente por la composición química de las hojas así como por la falta de humedad, la baja temperatura y el $\mathrm{pH}$ ácido, lo que puede modificar algunos factores microambientales al actuar como un filtro físico (Vellend et al. 2000), que impide que algunas semillas se almacenen en el suelo y formen parte del banco de semillas. Aunado a esto, al modificar algunos factores abióticos (luz, temperatura, y precipitación entre otros) y del suelo (cantidad y calidad de la hojarasca, humedad y compactación) (Molofsky \& Augspurger 1992) podrían afectar la germinación. Por lo que resulta de gran importancia conocer cómo actúa la hojarasca como filtro físico y considerar los atributos de las especies, ya que de ellos depende en buena parte, su capacidad de respuesta ante las barreras o "filtros" (Vellend et al. 2000).

En este trabajo a través de la composición y abundancia del banco de semillas y la vegetación establecida se identificaron los grupos funcionales que los componen bajo diferentes factores abióticos y de disturbio antropogénico en laderas orientadas al sur y laderas orientadas al norte del bosque de Quercus rugosa de la cuenca del río Magdalena (CRM), así como comparar la composición de las especies 
presentes en el banco de semillas del suelo con el de la hojarasca. Nuestras preguntas de investigación fueron ¿Qué factores abióticos determinan la germinación de nuevos individuos de distintos grupos funcionales? y ¿De qué manera esta información puede contribuir en la planeación de programas de conservación y restauración? Por lo que, nuestra hipótesis predice, que sí los factores abióticos (temporales y espaciales) determinan el ensamblaje de las especies presentes en la vegetación y el banco de semillas, estos actuarán como filtros de selección en la formación de grupos funcionales de especies. Dado que los filtros abióticos son más restrictivos en la temporada seca, se espera que la similitud de especies entre el banco de semillas y la vegetación en pie sea diferente entre temporadas. Además, debido a que el bosque bajo estudio es continuamente afectado por disturbios antropogénicos, la similitud de especies entre el banco de semillas y la vegetación en pie sea mayor a $50 \%$, por la presencia de especies de la vegetación secundaria, herbáceas y pioneras que conforman grupos funcionales característicos de etapas sucesiones tempranas. Esto podría, aportar información valiosa sobre la importancia que tienen los filtros en la dinámica del banco de semillas y contribuir a entender el papel de los grupos funcionales de especies que germinan en diferentes sustratos.

\section{Materiales y métodos}

Sitio de estudio. La cuenca del río Magdalena (CRM) se

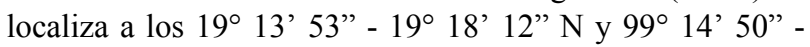
$99^{\circ} 20^{\prime} 30^{\prime}$ O, forma parte de la Sierra de las Cruces, en el Eje Volcánico Transversal (Álvarez-Román 2000). Se localiza al límite suroccidental de la ciudad de México. Tiene una extensión de 2,925 ha (Ávila-Akerberg 2002) y su relieve montañoso va de los $2,470 \mathrm{~m} \mathrm{snm}$. en el noreste a los 3,850 m snm al suroeste. La vegetación está compuesta por tres tipos de bosque: bosque de Pinus hartwegii Lind. en las partes más altas; bosque de Abies religiosa (Kunth) Schltdl. \& Cham., en la parte media; en las partes más bajas bosque Quercus rugosa Née. La CRM muestra diferentes actividades de disturbio antrópico recurrente, como ganadería, deforestación y deshierbe (chaponeo), que han promovido la pérdida de la biodiversidad y el incremento de la colonización de las especies introducidas (Martínez-Orea et al. 2019).

En el bosque de Q. rugosa se establecieron 15 parcelas al azar orientadas en la ladera norte y 15 orientadas en la ladera sur, todas de $5 \times 5 \mathrm{~m}$, donde se registró tanto en la temporada de secas (noviembre-mayo 2012) como en la de lluvias (junio-octubre 2013) la riqueza y abundancia de las especies de la vegetación establecida (VE), tomando en cuenta, las especies herbáceas y arbustivas con una altura menor a $3 \mathrm{~m}$. Para determinar la riqueza y abundancia de las especies del banco de semillas (cuantificado a partir de las plántulas que emergen de los sustratos), en cada parcela se colectaron tres muestras de 300 g por cada uno de los sustratos determinados 1) hojarasca grande (HG) definida como los residuos sin descomponer, 2) hojarasca chica (Hch) definido como los residuos y hojas parcialmente descompuesta con presencia de materia orgánica (mantillo) y 3) suelo (Suelo) definido como el sustrato proveniente del primer horizonte mineral del suelo.

Las muestras de cada sustrato se colocaron en macetas de germinación de $30 \times 15 \times 7 \mathrm{~cm}$, teniendo un total de 270 muestras independientes por temporada. En cada maceta se colocó una capa de $2 \mathrm{~cm}$ de profundidad de vermiculita sobre la cual se depositó la muestra para disminuir la pérdida de humedad. Las macetas se mantuvieron durante el año de estudio, en un vivero cerrado (30\% de condiciones de sombra) para evitar la contaminación de las muestras y cercano al sitio de estudio $2,500 \mathrm{~m} \mathrm{snm}$. temperatura promedio de $17^{\circ} \mathrm{C}$. Se registró la emergencia de plántulas quincenalmente durante un año, las plántulas emergidas se trasplantaron para permitir su crecimiento y posterior identificación.

En cada parcela se registró la orientación de la ladera (norte, sur). Durante cada temporada (lluvias y secas) se registraron los siguientes factores abióticos: luz y factores químicos del suelo. La luz se cuantificó a través de fotografías hemisféricas, las cuales fueron tomadas en cada parcela con un lente hemisférico y se analizaron con el software HemiView Canopy Image Analysis System, v.2.1 (Delta-T Devices 1998-1999), para estimar el factor de sitio global FSG $\left(\mathrm{GSF}, \mathrm{MJ} / \mathrm{m}^{-2} / \mathrm{yr}^{-1}\right)$. También se realizó un análisis químico del suelo para conocer el $\mathrm{pH}$, porcentaje de materia orgánica $(\mathrm{MO})$, nitrógeno $(\mathrm{N})$, potasio total $(\mathrm{K})$ conductividad eléctrica $(\mathrm{CE})$ y fósforo disponible $(\mathrm{P})$.

Las especies se clasificaron en tres categorías: introducidas, malezas y características. Las introducidas fueron las especies que tienen un origen biogeográfico diferente a México; las malezas fueron aquellas especies que originalmente evolucionaron en nuestro país y que son comunes en sitios que están perturbados por actividades antropogénicas; las especies características fueron plantas nativas que se originaron en nuestro país y forman parte de la flora de bosques templados (Calderón de Rzedowski \& Rzedowski 2005).

Análisis de datos. Para conocer el efecto temporal y espacial sobre la vegetación establecida (VE) y el banco de semillas (BS), se realizó un análisis de conglomerados mediante el método divisivo de dos vías (TWINSPAN), tomando en cuenta el porcentaje de similitud de acuerdo con el índice de Sørensen con el programa PC-ORD, ver. 7 (Peck 2016). En el análisis se clasificaron a las especies considerando el número de plántulas $\mathrm{y}$ de individuos por especie 
(abundancia absoluta) con relación a las parcelas (variación espacial; orientación de la ladera y temporal; temporada de seca y lluvia) (Hill 1979). Las especies que formaron una agrupación mayor al $50 \%$ se definieron como grupos funcionales distintos. La representación gráfica de la relación de los grupos funcionales se realizó con el software

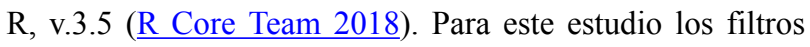
ambientales son determinados con base en los cambios temporales (secas y lluvias) y espaciales (orientación de la ladera, hojarasca chica, hojarasca grande y suelo) a través de los cuales se modifican las condiciones de luz y las propiedades químicas del suelo. Autores como Egan \& Ungar (2000), proponen la agrupación en conjunto de especies del banco de semillas y de la vegetación establecida para determinar grupos funcionales.

Posteriormente se realizó un procedimiento de Permutación de Respuesta Múltiple (MRPP) para examinar la disimilitud de especie entre y dentro de los grupos funcionales determinados, usando como medida de disimilitud el índice de Sørensen. El análisis MRPP es un procedimiento no paramétrico, ampliamente utilizado en los estudios de vegetación y de banco de semillas que evalúa las diferencias de composición de especies entre grupos basados en las matrices de disimilitud (Anderson \& Walsh 2013). Para determinar si los valores de disimilitud entre grupos funcionales dependen de la escala espacial (orientación de la ladera) o temporal (temporada de seca y de lluvia), para cada una se realizó un análisis de varianza de permutación multivariado (PERMANOVA), usando 999 permutaciones Montecarlo aleatorias mediante el paquete estadístico de Vegan y RVAideMemoire con el R software, v. 3.5. El análisis de PERMANOVA se basa en el cálculo previo de la distancia entre dos objetos incluidos (similitud), basándose en la hipótesis nula de que la dispersión de los grupos con respecto al centroide son equivalentes para todos los grupos, por lo que el análisis prueba el efecto de factores en la separación de grupos en el espacio multidimensional de especies. PERMANOVA se basa en la prueba estadística de $\mathrm{T}$ y explica la significancia de separación entre grupos a través del valor de $P$ (Anderson \& Walsh 2013). Por último, con el fin de analizar la relación entre los factores abióticos y los grupos funcionales definidos se realizó un análisis multivariado de correspondencia canónica (CCA), realizado a través del software PC-ORD v.7 (Peck 2016).

\section{Resultados}

Similitud de especies entre banco de semillas (BS) y la vegetación establecida (VE). La vegetación establecida registró un total de 51 especies en la temporada seca y 58 especies en la temporada de lluvia. Respecto a su abundancia, se encontraron 684 individuos en los $750 \mathrm{~m}^{2}$ muestreados en ambas temporadas para la vegetación establecida (VE), mientras que en el banco de semillas (BS) se registró un total de 30 especies en secas y 22 especies en lluvias con una abundancia de 1,942 plántulas en total (Tabla 2).

Al comparar los índices de similitud entre los diferentes sustratos con la VE, se observó una mayor proporción de valores intermedios que oscilan entre los 0.4-0.6. De acuerdo a lo esperado la similitud entre la VE de la temporada de lluvias con el BS del sustrato HG de la temporada de lluvias fue bajo (índice de similitud $=0.194$ ). Se observó que la mayor similitud fue entre el BS en el sustrato suelo (Suelo) en la temporada de lluvias con el BS en el sustrato Hch en la misma temporada (índice de similitud $=0.811)(\underline{\text { Tabla 1 }}, \underline{\text { Figura 1 }})$.

Tabla 1. Proporción de similitud entre los diferentes estratos del BS y de la VE. Vegetación establecida en lluvias $=$ VE-Ll, en secas $=$ VE-Se. Banco de semillas en suelo y lluvias = Suelo-L1, en suelo y secas = Suelo-Se, en hojarasca grande y lluvias = HG-L1, en hojarasca grande y secas $=$ HG-Se, en hojarasca chica en lluvias $=$ Hch-Ll, y en hojarasca chica en secas $=$ Hch-Se

\begin{tabular}{ccccccccc}
\hline \multicolumn{7}{c}{ VE-LI } & VE-Se & Suelo-LI Suelo-Se HG-LI HG-Se Hch-LI Hch-Se \\
\hline VE-LI & 1 & & & & & & \\
VE-Se & 0.462 & 1 & & & & & \\
Suelo-Ll & 0.375 & 0.458 & 1 & & & & \\
Suelo-Se & 0.423 & 0.462 & 0.792 & 1 & & & & \\
HG-Ll & 0.194 & 0.258 & 0.370 & 0.258 & 1 & & \\
HG-Se & 0.300 & 0.350 & 0.556 & 0.600 & 0.316 & 1 & & \\
Hch-Ll & 0.390 & 0.390 & 0.811 & 0.683 & 0.400 & 0.552 & 1 & \\
Hch-Se & 0.531 & 0.531 & 0.800 & 0.776 & 0.286 & 0.595 & 0.789 & 1 \\
\hline
\end{tabular}


[

Martínez-Orea et al. / Botanical Sciences 98(3): 487-499. 2020

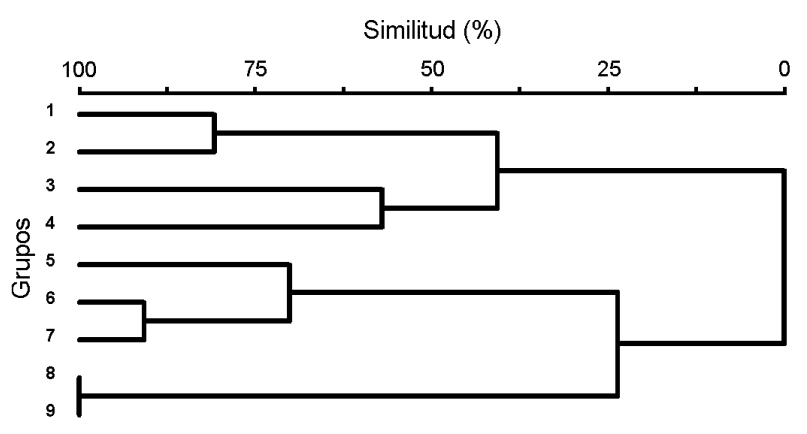

Figura 1. Agrupación basada en el índice de similitud de Sørensen.

Grupos funcionales. En el análisis de clasificación (TWINSPAN) se determinaron nueve grupos funcionales de especies entre los tres sustratos del BS y la VE (Figura 1, Tabla 2). A continuación, se describen las características de cada uno:

Grupo 1.- Grupo que caracteriza la VE, asociado a la ladera norte tanto en lluvias como en secas, conformado por 23 especies donde Salvia mexicana, Clematis dioica, Piqueria trinervia y Senecio angulifolius fueron las especies más abundantes. 11 especies son malezas nativas y las restantes son especies características del bosque templado. Así mismo, lo integran herbáceas y arbustivas perennes, donde la barocoria y la anemocoria son los síndromes característicos de este grupo. Especies como Bouvardia ternifolia, Brickellia pendula y Valeriana clematitis. Estuvieron ausentes en el BS y presentes en la VE.

Grupo 2.- Grupo que caracteriza la VE y al sustrato Suelo, asociado a la ladera sur, tanto en secas como en lluvias, pero también se encuentra en la ladera norte, aunque con menores abundancias. Es el grupo más heterogéneo conformado por 42 especies donde Gamochaeta americana, Oxalis corniculata, Salvia gesnerifolia, y Solanun cervantesii, malezas nativas, así como Hedera helix y Plantago major especies introducidas. Es un grupo conformado por herbáceas perennes y arbustos, principalmente con síndromes de dispersión por anemocoria, barocoria y endozoocoria.

Grupo 3.- Grupo que caracteriza al sustrato Hch, asociado a la ladera sur en la temporada de secas. Conformado por 18 especies donde Buddleia cordata árbol nativo de la vegetación secundaria es la especie con mayor abundancia, integrado por especies de forma de crecimiento herbáceo donde el síndrome de dispersión representativo fue la anemocoria. También en este grupo se presentó Plantago major, una especie introducida.

Grupo 4.- Grupo que caracteriza al sustrato Suelo y al sustrato Hch, asociado principalmente en la ladera norte tanto en lluvias como en secas, aunque también se presentó en menor cantidad en la ladera sur. Conformado por 11 especies donde Phytolacca icosandra arbusto perenne fue la especie con mayor abundancia, es un grupo conformado por todas las formas de crecimiento donde siete son malezas y una especie introducida. Los síndromes de dispersión relevantes son anemocoria y la barocoria.

Grupo 5.- Grupo asociado al sustrato Suelo, característico de las laderas sur, en ambas temporadas. Está conformado por 22 especies donde el arbusto perenne Archibaccharis serratifolia, presento la mayor abundancia. En este grupo también se encontraron otros arbustos con las mismas características, como Senecio angulifolius y Baccharis conferta, Este grupo presento nueve especies de malezas y dos especies introducidas, los síndromes de dispersión relevantes son la anemocoria y la endozoocoria.

Grupo 6.- Grupo presente en todos los sustratos del BS y en la VE tanto en lluvias como en secas en ambas laderas. Conformado por 16 especies donde Bromus carinatus fue la especie más abundante, la mayoría de las especies de este grupo son malezas herbáceas perennes donde Senecio sarguisobae fue exclusiva de este grupo, predominó el síndrome de dispersión de la barocoria, y en este grupo también se presentaron dos especies introducidas.

Grupo 7.- Grupo presente en todos los sustratos, principalmente en el sustrato Suelo y Hch, asociado a ambas laderas en ambas temporadas. Conformado por 25 especies donde Iresine diffusa, fue la más abundante, seguida de Senecio angulifolius, Linum usitatissimum esta última especie introducida. La mayoría de sus especies son perennes, siendo solamente tres especies anuales. Sigesbeckia jorullensis fue exclusiva de este grupo. Los síndromes de dispersión característicos de este grupo en orden de importancia fueron la anemocoria, la endozoocoria, la baracoria y la exozoocoria.

Grupo 8.- Es un grupo presente en todos los sustratos del BS sin especies de la VE. Representado principalmente en la temporada de secas en ambas laderas, aunque presente en las lluvias en la ladera norte. Conformado por 24 especies donde Solanum nigrescens $y$ Gamochaeta americana fueron las especies más abundantes, la mayoría de las especies son hierbas perennes, así como especies de otras formas de crecimiento en menores abundancias. Los síndromes de dispersión relevantes de este grupo fueron la endozoocoria, la anemocoria y la barocoria. En este grupo se presentaron tres especies introducidas con bajas abundancias. 
Grupo 9.- Es un grupo que caracteriza al sustrato Suelo, asociado a la ladera sur en la temporada de secas. Conformado por 15 especies donde Solanum nigrescens, hierba perenne y de dispersión endozoocora fue la más abundante. La mayoría de las especies que lo conforman presentaron abundancias bajas. En este grupo los síndromes de dispersión dominantes fueron la anemocoria y la barocoria. Destaca la ausencia de especies introducidas.

El análisis de permutación de respuesta múltiple, basado en los coeficientes de similitud de Sørensen, mostró una diferencia significativa de la composición entre los grupos funcionales $(\mathrm{A}=0.43 ; \mathrm{T}=-46.160 ; P<0.001)$. $\mathrm{La}$ similitud dentro de los grupos funcionales 1,9 y 8 mostraron los más altos valores del índice de similitud de composición de Sørensen, mientras que el grupo 2 mostró el menor valor del índice de similitud de composición (Figura 2).

Efecto ambiental sobre los grupos funcionales. El PERMANOVA indicó que la disimilitud de los grupos funcionales fue explicada de forma significativa por la temporada y la orientación $(P<0.005$, Tabla 1). Sin embargo, la interacción entre estos dos factores fue significativa (Tabla 3 $)$. Estos resultados demuestran que la temporada y la orientación de la ladera actúan de forma independiente como filtros ambientales de selección en las especies.

El análisis de correspondencias canónico entre los factores abióticos y los grupos funcionales, mediante la prueba de permutaciones de Monte Carlo, tuvo una representación significativa $(P<0.05$, Tabla 4$)$. De acuerdo con el CCA la luz está asociada positivamente con los grupos 5 y 6 , siendo esta una variable indicadora de la abundancia de los diferentes sustratos del BS. La altitud y la conductividad eléctrica del suelo mostraron una relación positiva con los grupos 3 y 7 , la cantidad de nitrógeno total, el fósforo disponible, el potasio total y el porcentaje de materia orgánica del suelo, así como la pendiente y la orientación de la ladera se encontraron asociados con los grupos 1, 4, 8 y 9. El pH se encontró asociado con el grupo 2 , mostró una tendencia significativa entre los factores abióticos y la distribución de los grupos (Figura 3).

\section{Discusión}

El ensamblaje de una comunidad depende de factores bióticos y abióticos, los cuales pueden actuar como filtros, limitando o facilitando la germinación y el establecimiento de las especies en un ecosistema (Booth \& Larson 1999). Este estudio apoya lo anterior ya que, independientemente de los atributos funcionales de cada especie para que sus semillas germinen y sus individuos sean reclutados; los factores abióticos del sitio son decisivos en la dinámica del banco de semillas en la comunidad. La liberación y reciclaje de nutrientes como producto de la descomposición de la materia orgánica y la hojarasca afectan directamente a la vegetación establecida (Wardle et al. 2002).

De acuerdo con el índice de similitud de Sørensen menos del $50 \%$ de las especies son compartidas entre el banco de semillas y la flora establecida. En un estudio previo se reportó una similitud de $31 \%$ entre ambos componentes en bosques afectados por la roza, tumba y quema (MartínezOrea et al. 2013). El bosque de encinos del sitio de estudio es constantemente afectado por la urbanización (ya que está inmerso en la Ciudad de México) y la ganadería, lo que puede explicar la alta similitud ( $>50 \%$ ) reportada en el banco de semillas del sustrato hojarasca chica (Hch) para la temporada seca. Este resultado podría deberse a la fenología reproductiva de las especies que dispersan sus semillas principalmente en la temporada seca (Martínez-Orea et al. 2013, Bonilla-Valencia et al. 2017), integrándose en el sustrato Hch. Por lo tanto, la temporalidad tuvo un efecto significativo sobre la riqueza y abundancia de especies en los grupos funcionales que caracterizaron este sustrato en el análisis.

Los grupos funcionales estuvieron representados por las diferencias de abundancia en las especies. Los grupos 1 y 2 caracterizan a la flora, los cuales presentaron una respuesta significativa a la temporalidad y a la orientación de la ladera. Esto podría sugerir que estos grupos de especies están formados principalmente por pioneras con ciclos de vida cortos (Baker 1974), cuya germinación y establecimiento se ven favorecidos en condiciones de disturbios frecuentes (Martínez-Orea et al. 2013). Aunado a esto, los factores abióticos de los sitios con mayor frecuencia de disturbios antrópicos pueden ser adecuados para el establecimiento de especies de sitios perturbados, con alta plasticidad fenotípica y tolerancia al estrés. La germinación de estas semillas puede presentar mayores porcentajes en sitios con mayor incidencia de luz y mayor temperatura (Radosevich et al. 2007).

En las laderas orientadas al sur la cantidad de luz que incide en los sitios es mayor (Méndez-Toribio et al. 2016). Además, es en estos sitios donde el dosel es más abierto y por lo tanto el porcentaje total de luz que incide es también mayor que en las parcelas con orientación norte. Este factor fue determinante para la abundancia de algunas especies que caracterizaron algunos grupos del banco en sus diferentes sustratos como Archibaccharis serratifolia (Kunth) S.F.Blake, Iresine diffusa Humb. \& Bonpl. ex Willd. y Physalis coztomatl Dunal, las cuales están catalogadas como malezas y germinan en condiciones de luz y temperaturas cálidas (Baskin \& Baskin 2004, Calderón de Rzedowski \& Rzedowski 2005). Baccharis conferta Kunth es una especie pionera que crece bajo claros del dosel en bosques de Abies religiosa (Cornejo-Tenorio et 
Tabla 2. Caracterización de los nueve grupos funcionales en los diferentes sustratos del BS y la VE del bosque de Quercus rugosa, se presentan las abundancias de las especies con mayores valores en los grupos caracterizados. Para la temporada de lluvia y seca se indica las muestras en la cara norte/sur. En color rojo se indica las especies malezas introducidas. Forma de crecimiento (FC): (h) hierba, (at) arbusto trepador, (ar) arbusto, (a) árbol; Ciclo de vida (CV): (an) anual, (p) perenne; Síndrome de dispersión (SD): (end) endozoocoria, (bar) barocoria, (ane) anemocoria, (exo) exozoocoria; Estatus (ES): (c) característica de bosque, (m) maleza nativa, (i) introducida.

\begin{tabular}{|c|c|c|c|c|c|c|c|c|c|c|c|c|c|}
\hline \multirow[b]{2}{*}{ Número de individuos } & \multicolumn{9}{|c|}{ Grupos } & & & & \\
\hline & 1 & 2 & 3 & 4 & 5 & 6 & 7 & 8 & 9 & 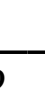 & & & \\
\hline Vegetación establecida (VE) & 358 & 253 & 5 & 2 & 0 & 37 & 39 & 0 & 0 & & & & \\
\hline Sustrato suelo (Suelo) & 0 & 98 & 13 & 31 & 184 & 3 & 185 & 165 & 145 & & & & \\
\hline Sustrato hojarasca chica (Hch) & 0 & 23 & 82 & 17 & 52 & 30 & 122 & 23 & 0 & & & & \\
\hline Sustrato hojarasca grande (HG) & 0 & 2 & 28 & 1 & 0 & 18 & 17 & 19 & 0 & & & & \\
\hline Abundancia total & 358 & 376 & 128 & 51 & 236 & 88 & 363 & 207 & 145 & & & & \\
\hline \multicolumn{14}{|l|}{ Número de especies } \\
\hline Malezas & 11 & 13 & 8 & 7 & 9 & 9 & 11 & 10 & 8 & & & & \\
\hline Introducidas & 0 & 4 & 1 & 1 & 2 & 1 & 2 & 2 & 0 & & & & \\
\hline Características & 12 & 25 & 9 & 3 & 11 & 6 & 12 & 12 & 7 & & & & \\
\hline Número total de especies & 23 & 42 & 18 & 11 & 22 & 16 & 25 & 24 & 15 & & & & \\
\hline Número de muestras & 25 & 33 & 14 & 13 & 15 & 17 & 30 & 31 & 9 & & & & \\
\hline Muestras en lluvias norte/sur & $10 / 1$ & $7 / 12$ & $2 / 2$ & $3 / 1$ & $0 / 5$ & $2 / 7$ & $4 / 7$ & $8 / 2$ & $2 / 1$ & & & & \\
\hline Muestras en secas norte/sur & $13 / 1$ & $5 / 9$ & $4 / 6$ & $7 / 2$ & $0 / 10$ & $4 / 4$ & $14 / 5$ & $11 / 10$ & $1 / 4$ & & & & \\
\hline Abundancias & & & & & & & & & & FC & $\mathrm{CV}$ & SD & $\mathrm{ES}$ \\
\hline Salvia mexicana $\mathrm{L}$. & 163 & 25 & 0 & 0 & 0 & 0 & 0 & 0 & 0 & $\mathrm{a}$ & $\mathrm{p}$ & bar & $\mathrm{r} \mathrm{m}$ \\
\hline Gamochaeta americana (Mill.) Wedd. & 0 & 30 & 4 & 2 & 6 & 0 & 1 & 47 & 8 & $\mathrm{~h}$ & $\mathrm{a}$ & ane & $\mathrm{e} \mathrm{m}$ \\
\hline Buddleia cordata Kunth & 1 & 2 & 84 & 0 & 0 & 0 & 1 & 2 & 1 & ar & $\mathrm{p}$ & ane & e c \\
\hline Phytolacca icosandra Sims. & 4 & 3 & 4 & 29 & 7 & 3 & 18 & 9 & 2 & a & $\mathrm{p}$ & end & $\mathrm{d} m$ \\
\hline Archibacaris serratifolia (Kunth) S.F.Blake & 0 & 0 & 3 & 1 & 111 & 2 & 10 & 6 & 1 & a & $\mathrm{p}$ & ane & $\mathrm{e}$ \\
\hline Bromus carinatus Hook. et Arn. & 1 & 8 & 3 & 2 & 5 & 38 & 4 & 12 & 0 & $\mathrm{~h}$ & $\mathrm{p}$ & bar & $\mathrm{r} \mathrm{m}$ \\
\hline Iresine diffusa Humb. et Bonpl. ex Willd. & 1 & 2 & 0 & 2 & 3 & 0 & 137 & 2 & 0 & $\mathrm{~h}$ & $\mathrm{p}$ & ane & $\mathrm{e} \mathrm{m}$ \\
\hline Senecio angulifolius DC. & 20 & 17 & 2 & 3 & 27 & 0 & 61 & 0 & 1 & a & $\mathrm{p}$ & ane & e c \\
\hline Solanum nigrescens M.Martens et Galeotti & 0 & 2 & 7 & 3 & 16 & 1 & 19 & 55 & 96 & a & $\mathrm{p}$ & end & $\mathrm{d} m$ \\
\hline Linum usitatissimum $\mathrm{L}$. & 0 & 2 & 0 & 2 & 3 & 2 & 54 & 2 & 0 & $\mathrm{~h}$ & $\mathrm{a}$ & bar & $r \quad i$ \\
\hline Clematis dioica $\mathbf{L}$. & 29 & 4 & 3 & 2 & 2 & 6 & 13 & 4 & 2 & at & $\mathrm{p}$ & ane & $\mathrm{e} m$ \\
\hline Piqueria trinervia Cav. & 24 & 19 & 0 & 0 & 0 & 0 & 2 & 0 & 0 & $\mathrm{~h}$ & $\mathrm{p}$ & ane & $\mathrm{e} m$ \\
\hline Oxalis corniculata $\mathbf{L}$. & 2 & 27 & 4 & 0 & 1 & 7 & 0 & 5 & 0 & $\mathrm{~h}$ & $\mathrm{p}$ & ane & $\mathrm{e} m$ \\
\hline Salvia gesneriflora Lindl. et Paxton & 2 & 25 & 0 & 0 & 0 & 4 & 2 & 0 & 0 & $\mathrm{~h}$ & $\mathrm{p}$ & bar & $\mathrm{r} \mathrm{m}$ \\
\hline Solanum cervantesii Lag. & 8 & 22 & 1 & 2 & 6 & 1 & 3 & 12 & 2 & $\mathrm{a}$ & $\mathrm{p}$ & end & d c \\
\hline Hedera helix $\mathrm{L}$. & 0 & 21 & 0 & 0 & 0 & 2 & 0 & 0 & 0 & at & $\mathrm{p}$ & end & d i \\
\hline Prunus serotina (Cav.) McVaugh & 0 & 20 & 5 & 0 & 1 & 4 & 3 & 2 & 0 & ar & $\mathrm{p}$ & end & d c \\
\hline Physalis coztomatl Moc. et Sessé ex Dunal & 0 & 1 & 0 & 1 & 19 & 0 & 5 & 4 & 0 & a & $\mathrm{p}$ & end & d c \\
\hline Fragaria mexicana Schltdl. & 0 & 3 & 1 & 0 & 2 & 0 & 7 & 14 & 3 & $\mathrm{~h}$ & $\mathrm{p}$ & end & d c \\
\hline Alchemilla procumbens Rose & 1 & 26 & 1 & 0 & 1 & 1 & 4 & 12 & 0 & $\mathrm{~h}$ & $\mathrm{p}$ & bar & $\mathrm{r} \mathrm{m}$ \\
\hline Roldana barba-johannis (DC.) H.Rob. et Brettell & 10 & 9 & 1 & 0 & 3 & 2 & 0 & 0 & 0 & a & $\mathrm{p}$ & ane & e c \\
\hline Salvia microphylla Kunth & 12 & 12 & 0 & 0 & 0 & 0 & 0 & 0 & 0 & a & $\mathrm{p}$ & bar & $\mathrm{r} \quad \mathrm{c}$ \\
\hline Acaena elongata $\mathrm{L}$. & 0 & 10 & 0 & 0 & 0 & 4 & 1 & 0 & 0 & a & $\mathrm{p}$ & exo & $\mathrm{om}$ \\
\hline Stachys repens M. Martens et Galeotti & 0 & 12 & 0 & 1 & 0 & 0 & 1 & 2 & 1 & $\mathrm{~h}$ & $\mathrm{p}$ & bar & $r \quad c$ \\
\hline Baccharis conferta Kunth & 0 & 0 & 0 & 0 & 15 & 0 & 0 & 0 & 0 & a & $\mathrm{p}$ & ane & e c \\
\hline Commelina coelestis Willd. & 4 & 10 & 0 & 0 & 0 & 0 & 0 & 0 & 0 & $\mathrm{~h}$ & $\mathrm{p}$ & bar & $\mathrm{r} \mathrm{m}$ \\
\hline Plantago major $\mathrm{L}$. & 0 & 14 & 1 & 0 & 0 & 0 & 0 & 3 & 0 & $\mathrm{~h}$ & $\mathrm{p}$ & ane & e i \\
\hline Senecio sanguisorbae DC. & 0 & 0 & 0 & 0 & 0 & 8 & 0 & 0 & 0 & $\mathrm{~h}$ & $\mathrm{p}$ & ane & e c \\
\hline Sonchus oleraceus L. & 0 & 1 & 0 & 0 & 2 & 0 & 6 & 0 & 0 & $\mathrm{~h}$ & $\mathrm{a}$ & ane & e i \\
\hline
\end{tabular}


al. 2003). Senecio barba-johannis (DC.) H.Rob. \& Brettell es una especie característica de la vegetación secundaria (Calderón de Rzedowski \& Rzedowski 2005). Sonchus oleraceus L. y Linum usitatissimum L. se han registrado en estudios anteriores en el bosque de Q. rugosa (CastroGutiérrez 2013), estas dos últimas son especies herbáceas, malezas introducidas de Europa y Asia, respectivamente, e intolerantes a la sombra, lo cual sugiere que la luz es un factor limitante para su emergencia desde el banco (Fenner 1987, Salonen \& Suhonen 1995). Además, S. oleraceus produce una gran cantidad de semillas (Chauhan et al. 2006), por lo que su aporte al banco es importante y dado que sólo una baja proporción de sus semillas presentan latencia innata (> $20 \%$ ), germina entre un 65 y un $100 \%$ después de ser dispersadas. Por otro lado, esta especie puede formar parte del aporte transitorio de los bancos de semillas que precisamente contiene semillas sin latencia (Baskin \& Baskin 2004). Del mismo modo L. usitatissimum, también se registró como parte de un componente transitorio pues su germinación rápida después de su integración al suelo permite suponer que carece de latencia (Dexter et al. 2011), por lo que formaría parte de un banco de semillas transitorio en el sitio de estudio.

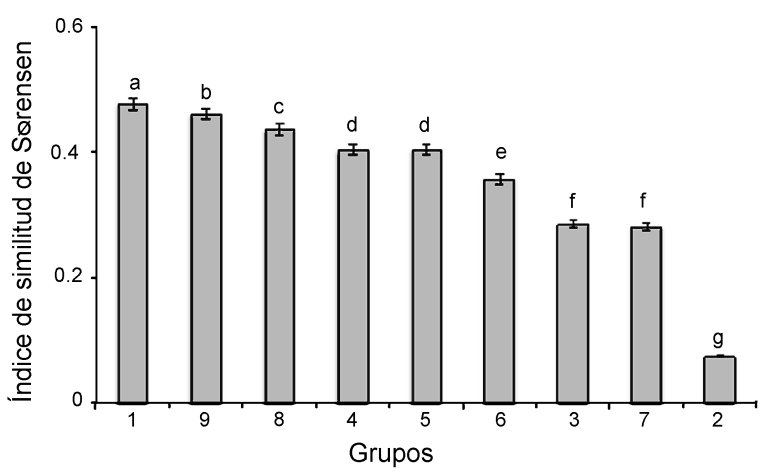

Figura 2. Valores del índice de similitud de Sørensen obtenidos para la comparación de la composición de especies entre grupos. Las barras de error son intervalos de confianza de $95 \%$. Las letras diferentes indican diferencias significativas en $P<0.001$.

Es importante mencionar que en todos los grupos la composición de especies que caracterizaron los sustratos estuvo representada por especies catalogadas como malezas nativas. Por ejemplo, Baccharis conferta no crece bajo un dosel cerrado y define al grupo 5 que está conformado por especies que solo se encontraron en la ladera sur. La intolerancia a la sombra y el fotoblastismo positivo (germinación solo bajo incidencia de luz) son características de las especies con semillas pequeñas, que por lo general pueden llegar a partes profundas del suelo (Arévalo \& Fernández-Palacios 2000), lo que explica que los diferentes sustratos pueden actuar como filtros para la permanencia/ emergencia de las semillas en el banco.
Tabla 3. Análisis PERMANOVA de los índices de similitud de Sørensen con los factores temporada y orientación de ladera. $\mathrm{x}=$ interacción. $*$ diferencias significativas. d.f. $=$ grados de libertar, SS = suma de cuadrados, MS = media de cuadrados, $R^{2}=$ varianza explicada por los factores, $P=$ valor de probabilidad

\begin{tabular}{lcccccc}
\hline & d.f. & SS & MS & $\boldsymbol{F}$ & $\boldsymbol{R}^{2}$ & $\boldsymbol{P}$ \\
\hline Temporada & 1 & 0.981 & 0.981 & 2.348 & $0.012<0.005^{*}$ \\
Orientación ladera & 1 & 3.145 & 3.144 & 7.525 & $0.038<0.005^{*}$ \\
$\begin{array}{l}\text { Temporada x } \\
\text { Orientación ladera }\end{array}$ & 2 & 0.571 & 0.570 & 1.365 & 0.006 & 0.138 \\
Residual & 183 & 76.43 & 0.417 & & 0.94 & \\
\hline
\end{tabular}

El sustrato Suelo presentó la mayor riqueza y abundancia de especies de los diferentes grupos funcionales. Senecio angulifolius D.C. y Solanum nigrum M.Martens et Galeotti son especies que tienen poblaciones de semillas que pueden germinar en condiciones de luz rojo lejano (Taab \& Andersson 2009), las cuales se encuentran bajo un dosel cerrado (Górski et al. 1978). Estas especies pueden permanecer viables mientras están enterradas, hasta que ocurre un disturbio, se ha reportado que sus tiempos de viabilidad se pueden extender hasta por dos años, aunado al hecho de que $S$. angulifolius ha mostrado porcentajes de germinación cercanos al 50\% en condiciones de luz rojo lejano (Martínez-Orea et al. 2019). Para el bosque estudiado estas especies fueron características de los grupos 7 y 8 , respectivamente. Además, los grupos 7 y 8 tuvieron altas abundancias en laderas al sur en los sustratos Suelo y Hch, y de acuerdo con Baskin y Baskin (2004) podrían formar parte del aporte persistente del banco de semillas. Por otro lado, algunas especies de los géneros Senecio y Solanum pueden tener una proporción alta de semillas con latencia fisiológica y latencia innata respectivamente y también se les ha encontrado en otras fuentes de la regeneración natural como la lluvia de semillas de este bosque (Ren \& Abbott 1991, Commander \& Kollo 2008, Martínez-Orea et al. 2013).

Tabla 4. Resultados de la prueba de permutaciones de Montecarlo realizadas para el análisis de correspondencias canónico.

\begin{tabular}{cccccc}
\hline Ejes & Eigen valores & Media & Mínimo & Máximo & $\boldsymbol{P}$ \\
\hline $\mathbf{1}$ & 0.239 & 0.131 & 0.073 & 0.231 & 0.0010 \\
$\mathbf{2}$ & 0.110 & 0.088 & 0.044 & 0.141 & \\
$\mathbf{3}$ & 0.076 & 0.062 & 0.032 & 0.109 & \\
\hline
\end{tabular}

Por lo general la composición del banco de semillas en bosques templados carece de especies arbóreas (Kjellsson 1992), sin embargo, reportamos a Prunus serotina McVaugh, Buddleja cordata Kunth y Clethra mexicana 
DC., como componentes arbóreos en las laderas orientadas al norte. En estos sitios la temperatura y sobre todo la humedad podrían ser los factores que determinan su presencia en los grupos 2 y 3 con abundancias intermedias en el BS sustrato Suelo y de la VE. Las plántulas de $P$. serotina y $B$. cordata únicamente se registraron en la temporada de lluvias, lo cual sugiere que requieren de niveles de humedad altos. Esto explica que estos grupos dependen del microclima más húmedo y menos caluroso que se genera gracias a la orientación de la ladera (Nevo et al. 1999).

Phartyal et al. (2009) reportaron a $P$. serotina como una especie que puede germinar en diferentes sustratos, como en nuestro estudio, por lo que su germinación no está limitada por los filtros de los diferentes sustratos, sino por las condiciones del efecto de ladera. Por otro lado, estos autores mencionan que no sólo la temperatura tiene un efecto en su germinación, sino que ambientes muy húmedos favorecen su germinación, principalmente en sitios orientados al norte. Además, tanto $P$. serotina como $B$. cordata han sido ya reportadas como componentes importantes del banco de semillas para la zona de estudio (Martínez-Orea et al. $\underline{2013})$.

Mientras el microclima de las parcelas orientadas al norte puede favorecer a las especies arbóreas, las especies de malezas arbustivas y herbáceas podrían verse favorecidas en sitios con mayor incidencia de luz. Esto explicaría la presencia de los grupos donde especies como

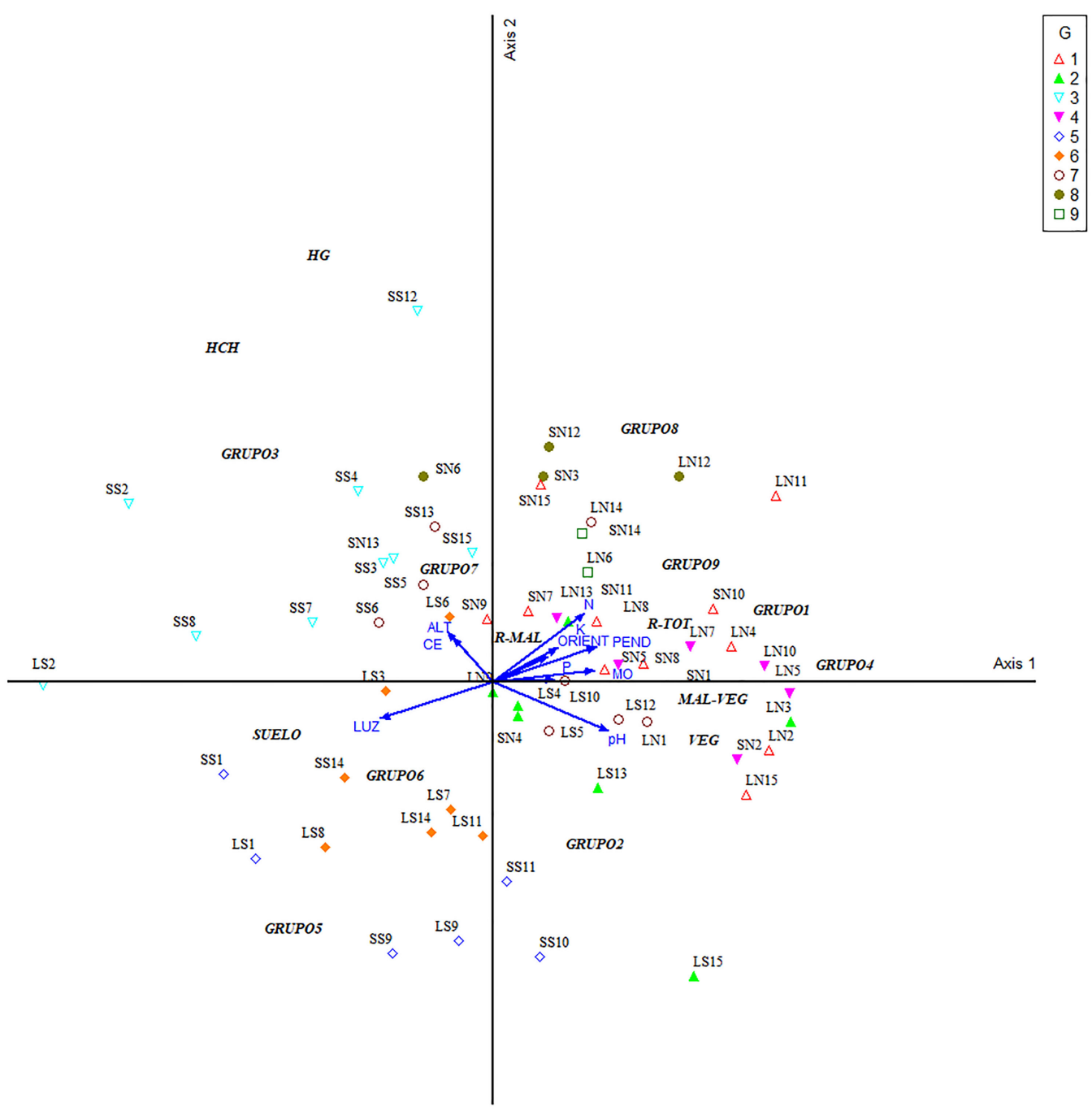

Figura 3. Análisis de Correspondencias Canónico que muestra la relación de los grupos funcionales (símbolos de colores = GRUPO 1 al 9 ) con los factores abióticos (flechas rojas) $\mathrm{N}=$ nitrógeno del suelo, $\mathrm{pH}=\mathrm{pH}$ del suelo, $\mathrm{MO}=$ materia orgánica del suelo, $\mathrm{CE}=$ conductividad eléctrica del suelo, $\mathrm{K}$ = potasio del suelo. $\mathrm{P}=$ fósforo del suelo. $\mathrm{R}$-malezas = riqueza de especies de malezas, malezas $\mathrm{VE}=$ número de malezas presentes en la vegetación establecida. Sustratos: Suelo, $\mathrm{HG}=$ hojarasca grande, $\mathrm{Hch}=$ hojarasca chica. Parcelas en secas $=\mathrm{S} n \mathrm{P} m$, parcelas en lluvias $=\mathrm{L} n \mathrm{P} m$, donde $n=1$ ubicadas en la orientación sur y $n=2$ ubicadas en la orientación norte y $m=$ número de parcela. 
A. serratifolia, Bouvardia ternifolia (Cav.) Schltdl y Oxalis corniculata L. son características en la ladera orientada al sur. Sin embargo, la humedad parece ser un factor determinante para su crecimiento, ya que solo se registraron para la flora durante la temporada de lluvias, al igual que las especies de malezas nativas como Senecio sanguisorbae DC., Bromus carinatus Hook. \& Arn. y Commelina coelestis Willd. Estas últimas especies también abundaron en los grupos caracterizados por su orientación al norte, con una incidencia de luz total del $26 \%$.

El grupo 2, caracterizado por Gamochaeta americana Mill., incluyó a Hedera helix L. (especie introducida, Calderón de Rzedowski \& Rzedowski 2005), que predominó en la flora del bosque de las laderas al norte. Esta especie podría verse favorecida por el microclima húmedo, el cual se asemeja al clima europeo de donde esta especie es originaria. El contenido de humedad en el suelo de las laderas al norte puede generar una mayor disponibilidad de nutrientes para las especies, tal puede ser el caso de la maleza nativa Salvia mexicana L., la cual fue la más abundante en el grupo 1 en las parcelas orientadas al norte.

Algunas características del suelo como el $\mathrm{pH}$ y el contenido de nitrógeno pueden también afectar la germinación y el establecimiento de las especies. El suelo de las laderas al norte contiene mayor porcentaje de materia orgánica, nitrógeno, potasio y fósforo que los suelos de laderas con orientación sur. El nitrógeno y el potasio son nutrientes esenciales, sobre todo durante etapas tempranas del crecimiento de las plántulas de muchas especies (Fenner 1987). En un estudio realizado por Mendoza-Hernández et al. (2014), se observó que la mortalidad de S. mexicana es baja y su crecimiento incrementa en micrositios húmedos. Llama la atención su ausencia en los diferentes sustratos del banco pues Jiménez-Hernández (2016) la reportó previamente para la zona de estudio.

Los resultados muestran que, dependiendo del tipo de sustrato en el que se encuentre la semilla, su germinación se ve favorecida o inhibida. El sustrato Suelo tuvo un efecto positivo sobre la germinación de las semillas, los sustratos de la hojarasca tuvieron un efecto negativo sobre algunas especies. Dado que la mayor riqueza y abundancia se presentó en el sustrato Suelo en todos los grupos y temporadas lo cual podría estar relacionado con la composición de especies. Así podemos ver que dentro de los grupos hubo una alta similitud, pero resultaron diferentes entre ellos, esto puede deberse por un lado a que la mayoría de los sitios de donde se tomaron las muestras presentó una capa muy gruesa (mayor a $30 \mathrm{~cm}$ ) de hojarasca proveniente principalmente de los árboles de Q. rugosa, sobre todo en aquellos orientados al norte. Por otro lado, hay parcelas donde la hojarasca está compuesta parcialmente por hojas de otros encinos (Quercus spp.) y de otras especies arbóreas, que incluyen a Garrya laurifolia Benth., $P$. serotina y $A$. religiosa, en algunas parcelas el suelo estaba pobremente cubierto por una capa de HG y el Suelo estaba parcialmente expuesto.

Los sustratos de hojarasca no fueron un impedimento para la entrada de semillas al banco del sustrato Suelo, ya que el número de plántulas emergidas fue mucho mayor en la mayoría de los grupos. Cabe resaltar que se presentaron diferencias significativas entre sustratos y temporadas. Las diferencias de abundancia entre los sustratos podrían estar relacionadas con la temporada de la dispersión de las semillas. Probablemente muchas de las especies que germinaron en la hojarasca dispersan sus semillas durante la temporada de secas, por lo que casi en todos los grupos se encontraron plántulas que germinaron en la hojarasca. Durante la temporada de lluvias se observó una drástica disminución en la riqueza y abundancia en las plántulas emergidas en la hojarasca, pero no en el suelo, ya que existe una mayor entrada de semillas al banco del sustrato Suelo durante la temporada de secas. Al llegar al Suelo, estas semillas pueden formar parte del banco de semillas y germinar en cuanto los factores abióticos sean los indicados para su reclutamiento y establecimiento en la vegetación, posiblemente durante la temporada de lluvias. Esto podría explicar el elevado número de plántulas que emergieron del suelo en esta temporada. Es importante considerar que las especies introducidas que se encuentran tanto en el banco como en la flora pueden desempeñarse como invasoras afectando a otras especies del sistema, por ejemplo, reduciendo la producción de semilla de las especies nativas, por lo que en algunos programas de restauración se ha optado por removerlas de la vegetación (Cook-Patton \& Agrawa 2014). Por lo anterior, sería adecuado mantener el monitoreo de esas especies en el sitio de estudio.

El presente estudio permite conocer que especies se encuentran formando parte del banco de semillas y de la flora en pie en un bosque afectado por disturbios antropogénicos. Esta información es útil porque permite saber cuáles especies pueden germinar desde diferentes sustratos del suelo y en que temporadas y es importante para conocer algunos de sus requerimientos para germinar, así como su disponibilidad como parte del potencial de regeneración natural bajo las presiones antropogénicas en el sitio. Por otro lado, debemos considerar que los resultados observados en vivero pueden subestimar las abundancias de algunas especies porque sus respuestas germinativas pueden ser distintas a las que se pudieran observar en el campo ( $\underline{\text { Ter }}$ Heerdt et al 1999, Brown 1992), principalmente debido a las diferencias en los factores de luz, temperatura y humedad entre estos dos sitios. Por esto es importante que los factores sean los adecuados para la germinación de la mayoría de las especies (Gross 1990), por lo que se decidió colocar el vivero muy cercano al área de estudio. Sin 
प

Martínez-Orea et al. / Botanical Sciences 98(3): 487-499. 2020

embargo, incluso en el campo la luz es un factor sumamente cambiante debido a la cobertura del dosel, la orientación de la ladera, la época del año, hora del día y la micro topografía, por lo que su variabilidad es alta incluso entre micrositios del bosque (Méndez-Toribio et al. 2016, Zhu et al. 2003). Por lo tanto, los estudios de la composición del banco de semillas realizados en condiciones de vivero tratan de capturar algo de la dinámica en las respuestas de las especies a su ambiente ya que experimentalmente no siempre es posible tener muestras de suelo en el campo, pues otros factores como la pérdida de las macetas, su contaminación con otras semillas en dispersión y/o su depredación son de difícil control en este caso.

En conclusión, el conocimiento de los factores que actúan como filtros para el establecimiento de diferentes grupos funcionales de especies, resulta de gran importancia en la conservación y restauración en este bosque a través del banco de semillas; mediante el cual, se determina la estructura de una comunidad e influye en la resiliencia ante los incrementos de abundancia de especies invasoras $\mathrm{o}$ indicadoras de perturbación en la vegetación establecida. Dentro de los grupos coexisten diferentes tipos de especies los cuales responden a los filtros abióticos y cambian tanto en la temporalidad como en la orientación lo cual tiene un efecto sobre la riqueza y abundancia de los grupos funcionales.

Dado que se encontraron especies nativas dentro de cada grupo funcional con diferentes estrategias de formación de banco de semillas, tales como las que germinan inmediatamente después de su dispersión y aquellas que permanecen latentes como parte de un banco permanente por lo que esto representa una ventaja para el potencial de regeneración natural.

\section{Agradecimientos}

Proyecto UNAM PAPIIT IN-211118. Proyecto UNAM PAPIME PE201817. A la comunidad de la Magdalena Contreras, Ciudad de México.

\section{Literatura citada}

Aide TM, Zimmerman JK, Pascarella JB, Rivera L, Marcano-Vega H. 2000. Forest regeneration in a chronosequence of tropical abandoned pastures: implications for restoration ecology. Restoration Ecology 8: 328-338. DOI: https://doi.org/10.1046/j.1526-100x.2 000.80048.x

Álvarez-Román KE. 2000. Geografía de la educación ambiental: algunas propuestas de trabajo en el bosque de los Dinamos, área de conservación ecológica de la Delegación Magdalena Contreras. BSc Thesis. Universidad Nacional Autónoma de México.
Anderson MJ, Walsh DCI. 2013. PERMANOVA, ANOSIM, and the Mantel test in the face of heterogeneous dispersions: what null hypothesis are you testing? Ecological monographs 83: 557-574. DOI: https://doi.org/10.1890/12-2010.1

Arévalo JR, Fernández-Palacios JM. 2000. Seed bank analysis of tree species in two stands of the Tenerife laurel forest (Canary Islands). Forest Ecology and Management 130: 177-185. DOI: https://doi.org/10.10 $\underline{\text { 16/S0378-1127(99)00182-6 }}$

Ávila-Akerberg VD. 2002. La vegetación de la cuenca alta del río Magdalena: un enfoque florístico, fitosociológico $y$ estructural. BSc. Thesis. Universidad Nacional Autónoma de México.

Baker HG. 1974. The evolution of weeds. Annual Review of Ecology and Systematics 5: 1-24. DOI: https://doi.org/ $\underline{\text { 10.1146/annurev.es.05.110174.000245 }}$

Baskin JM, Baskin CC. 2004. A classification system for seed dormancy. Seed Science Research 14: 1-16. DOI: https://doi.org/10.1079/SSR2003150

Bonilla-Valencia L, Martínez-Orea Y, Castillo-Argüero S, Barajas-Guzmán G, Romero-Romero MA, Díaz-López ET. 2017. Reproductive phenology of understory species in an Abies religiosa (Pinaceae) forest in the Magdalena River Basin, Mexico City. The Journal of the Torrey Botanical Society 144: 313-328. DOI: https://doi.org/ 10.3159/TORREY-D-16-00024.1

Booth BD, Larson DW. 1999. Impact of language, history, and choice of system on the study of assembly rules. In: Weiher E, P Keddy, eds. Ecological Assembly Rules: Perspectives, Advances, Retreats. pp. 206-229. Cambridge, United Kingdom: Cambridge University Press. ISBN: 9780521652353

Brown D. 1992. Estimating the composition of a forest seed bank: a comparison of the seed extraction and seedling emergence methods. Canadian Journal of Botany 70: 1603-1612. DOI: https://doi.org/10.1139/b92-202

Calderón de Rzedowski G, Rzedowski J. 2005. Flora fanerogámica del Valle de México. Pátzcuaro Michoacán: Comisión Nacional para el Conocimiento y Uso de la Biodiversidad e Instituto de Ecología, AC. ISBN; 978-607-7607-36-6

Castellanos-Castro C, Bonilla MA. 2011. Grupos funcionales de plantas con potencial uso para la restauración en bordes de avance de un bosque altoandino. Acta Biológica Colombiana 16: 153-174.

Castro-Gutiérrez C. 2013. El Papel de las especies invasoras en la estructura herbácea del bosque de Quercus rugosa, en la cuenca del Río Magdalena, D.F. BSc Thesis. Universidad Nacional Autónoma de México.

Catovsky S, Bazzaz FA. 2002. Nitrogen availability influences regeneration of temperate tree species in the understory seedling bank. Ecological Applications 12: 
Banco de semillas de un bosque de encino en la ciudad de México

प

1056-1070. DOI: https://doi.org/10.1890/1051-0761(20 02)012[1056:NAIROT]2.0.CO;2

Catovsky S, Bradford MA, Hector A. 2002. Biodiversity and ecosystem productivity: implications for carbon storage. Oikos 97: 443-448. DOI: https://doi.org/10.103 4/j.1600-0706.2002.970315.x

Chauhan BS, Gill G, Preston C. 2006. Factors affecting seed germination of annual sowthistle (Sonchus oleraceus) in southern Australia. Weed Science 54: 854-860. DOI: https://doi.org/10.1614/WS-06-047R.1

Cook-Patton SC, Agrawal AA. 2014. Exotic plants contribute positively to biodiversity functions but reduce native seed production and arthropod richness. Ecology 95, 1642-1650. DOI: https://doi.org/10.1890/13-0782.1

Commander S, Kollo J. 2008. The changing demand for skills Evidence from the transition. Economics of Transition and Institutional Change 16: 199-221. DOI: https://doi.org/10.1111/j.1468-0351.2008.00303.x

Cornejo-Tenorio G, Casas A, Farfán B, Villaseñor JL, Ibarra-Manríquez G. 2003. Flora y vegetación de las zonas núcleo de la Reserva de la Biósfera Mariposa Monarca, México. Boletín de la Sociedad Botánica de México. 73: 43-62. DOI: https://doi.org/10.17129/bot sci. 1678

Delta-T Devices. 1998-1999. User manual for HemiView. v.2.1. Cambridge, Reino Unido: Delta-T Devices. https:// www.delta-t.co.uk/wp-content/uploads/2017/02/hv2um2 1.2.pdf (acceso febrero, 2020).

Dexter JE, Jhala AJ, Yang RC, Hills MJ, Weselake RJ, Hall LM. 2011. Harvest loss and seed bank longevity of flax (Linum usitatissimum) implications for seed-mediated gene flow. Weed Science 59: 61-67. DOI: https://doi.org/ 10.1614/WS-D-10-00047.1

Dey DC, Knapp BO, Battaglia MA, Deal RL, Hart JL, O'Hara KL, Callie CJ, Schuler TM. 2019. Barriers to natural regeneration in temperate forests across the USA. New Forests 50: 11-40. DOI: https://doi.org/10.1007/ s11056-018-09694-6

Egan TP, Ungar IA. 2000. Similarity between seed banks and above-ground vegetation along a salinity gradient. Journal of Vegetation Science 11: 189-194. DOI: https:// doi.org/10.2307/3236798

Fenner M. 1987. Seedlings. New Phytologist 106: 35-47. DOI: https://doi.org/10.1111/j.1469-8137.1987.tb04681.x

Górski T, Górska K, Rybicki J. 1978. Studies on the germination of seeds under leaf canopy. Flora 167: 289-299. DOI: https://doi.org/10.1016/S0367-2530(17) 31121-0

Gross KL 1990. A comparison of methods for estimating seed numbers in the soil. Journal of Ecology 78:1079-1093. DOI: https://doi.org/10.2307/2260953

Hannah L, Carr JL, Lankerani A. 1995. Human disturbance and natural habitat a biome level analysis of a global data set. Biodiversity and Conservation 4: 128-155. DOI: https://doi.org/10.1007/BF00137781

Hill MO. 1979. TWINSPAN. A FORTRAN program for arranging multi-variate data in an ordered two-way table by classification of the individuals and attributes. Ithaca, Nueva York: Cornell University.

Jiménez-Hernández HA. 2016. Comparación del banco de semillas de dos zonas contrastantes del bosque de Quercus rugosa-Quercus laurina en la cuenca del Río Magdalena, D.F., México. BSc Thesis. Universidad Nacional Autónoma de México.

Kjellsson G. 1992. Seed banks in Danish deciduous forests: species composition, seed influx and distribution pattern in soil. Ecography 15: 86-100. DOI: https://doi.org/ 10.1111/j.1600-0587.1992.tb00012.x

Kostel-Hughes F, Young TP, Wehr JD. 2005. Effects of leaf litter depth on the emergence and seedling growth of deciduous forest tree species in relation toseed size. The Journal of the Torrey Botanical Society 132: 50-61. DOI: https://doi.org/10.3159/1095-5674(2005)132[50:EOLLD O]2.0.CO;2

Martínez-Orea Y, Castillo-Argüero S, Álvarez-Sánchez J, Collazo-Ortega M, Zavala-Hurtado JA. 2013. Lluvia y banco de semillas como facilitadores de la regeneración natural en un bosque templado de la ciudad de México. Interciencia 38: 400-409.

Martínez-Orea Y, Castillo-Argüero S, Orozco-Segovia A, Zavala-Hurtado JA, Bonilla-Valencia L. 2019. Micrositios seguros para la germinación de Symphoricarpos microphyllus (Caprifoliaceae), una especie arbustiva con latencia. Acta Botanica Mexicana 126: e1458. DOI: https://doi.org/10.21829/abm126.201 9.1458

Matus G, Tóthmérész B, Papp M. 2003. Restoration prospects of abandoned species-rich sandy grassland in Hungary. Applied Vegetation Science 6: 169-178. DOI: https://doi.org/10.1111/j.1654-109X.2003.tb00577.x

McKinney ML. 2002. Urbanization, biodiversity, and conservation: the impacts of urbanization on native species are poorly studied, but educating a highly urbanized human population about these impacts can greatly improve species conservation in all ecosystems. BioScience 52: 883-890. DOI: https://doi.org/10.1641/ 0006-3568(2002)052[0883:UBAC]2.0.CO;2

Méndez-Toribio M, Meave JA, Zermeño-Hernández I, Ibarra-Manríquez G. 2016. Effects of slope aspect and topographic position on environmental variables, disturbance regime and tree community attributes in a seasonal tropical dry forest. Journal of Vegetation Science 27: 1094-1103. DOI: https://doi.org/10.1111/ jvs. 12455

Mendoza-Hernández PE, Rosete-Rodríguez A, SánchezCoronado ME, Orozco S, Pedrero-López L, Méndez I, 
Orozco-Segovia A. 2014. Vegetation patches improve the establishment of Salvia mexicana seedlings by modifying microclimatic conditions. International Journal of Biometeorology 58: 853-866. DOI: https:// doi.org/10.1007/s00484-013-0665-8

Millar CI, Stephenson NL. 2015. Temperate forest health in an era of emerging megadisturbance. Science 349: 823-826. DOI: https://doi.org/10.1126/science.aaa9933

Molofsky J, Augspurger CK. 1992. The effect of leaf litter on early seedling establishment in a tropical forest. Ecology 73: 68-77. DOI: https://doi.org/10.2307/1938 $\underline{721}$

Nevo E, Fragman O, Dafni A, Beiles A. 1999. Biodiversity and interslope divergence of vascular plants caused by microclimatic differences at "Evolution Canyon" lower nahal Oren, Mount Carmel, Israel. Israel Journal Plant Sciences 47: 49-59. DOI: https://doi.org/10.1080/07929 978.1999.10676751

Pakeman RJ, Small JL. 2005. The role of the seed bank, seed rain and the timing of disturbance in gap regeneration. Journal of Vegetation Science 16: 121-130. DOI: https://doi.org/10.1111/j.1654-1103.2005.tb02345.x

Peck JE. 2016. Multivariate analysis for ecologists: step-bystep. 2a ed. Using PC-ORD v.7. Gleneden Beach, Oregon: MJM Software Design. https://www.wildb lueberrymedia.net/store/step-by-step (acceso febrero, 2020).

Phartyal SS, Godefroid S, Koedam N. 2009. Seed development and germination ecophysiology of the invasive tree Prunus serotina (Rosaceae) in a temperate forest in Western Europe. Plant Ecology 204: 285-294. DOI: https://doi.org/10.1007/s11258-009-9591-6

Primack RB, Kang H. 1989. Measuring fitness and natural selection in wild plant populations. Annual Review of Ecology and Systematics 20: 367-396. DOI: https:// doi.org/10.1146/annurev.es.20.110189.002055

R Core Team. 2018. R: a language and environment for statistical computing. $\mathrm{R}$ Foundation for Statistical Computing, Vienna, Austria. http://www.R-project.org/ (acceso febrero, 2020).

Radosevich SR, Holt JS, Ghersa CM. 2007. Ecology of Weeds and Invasive Plants: Relationship to Agriculture and Natural Resource Management. Hoboken, New Jersey: John Wiley \& Sons, Inc. ISBN: 9780471767794; DOI: $\underline{\text { https://doi.org/10.1002/9780470168943 }}$

Ren Z, Abbott RJ. 1991. Seed dormancy in Mediterranean Senecio vulgaris L. New Phytologist 117: 673-678. DOI: https://doi.org/10.1111/j.1469-8137.1991.tb00972.x

Salonen V, Suhonen J. 1995. Effects of seed weight on growth, reproduction and competitive ability of Linum

\footnotetext{
Editor de sección: Numa Pavón

Contribución de los autores: YMO escritura y redacción del texto; LBV estadística multivariada, escritura y redacción del texto; YVS escritura y redacción del texto; MARR redacción y formato del texto, diseño de tablas y figuras; SSO trabajo de campo y toma de datos y generación de matrices de datos; GSA colaboración en el análisis de datos; SCA escritura del texto diseño del estudio y análisis de estadística multivariada.
}

usitatissimum seedlings. Annales Botanici Fennici 32: 101-106.

Sánchez-Colón S, Flores-Martínez A, Cruz-Leyva IA, Velázquez A. 2009. Estado y transformación de los ecosistemas terrestres por causas humanas. In: CONABIO. Capital Natural de México, Vol. II: Estado de conservación y tendencias de cambio. Ciudad de México, Comisión Nacional sobre el Conocimiento y Uso de la Biodiversidad, pp. 75-129. ISBN: 978-607-7607-08-3

Taab A, Andersson L. 2009. Seasonal changes in seed dormancy of Solanum nigrum and Solanum physalifolium. Weed Research 49: 90-97. DOI: https:// doi.org/10.1111/j.1365-3180.2008.00674.x

Ter Heerdt GNJ, Schutter A, Bakker JP 1999. The effect of water supply on seed-bank analysis using the seedlingemergence method. Functional Ecology 13: 428-430. DOI: https://doi.org/10.1046/j.1365-2435.1999.00329.x

Tovar-Sánchez E, Cano-Santana Z, Oyama K. 2004. Canopy arthropod communities on Mexican oaks at sites with different disturbance regimes. Biological Conservation 115: 79-87. DOI: https://doi.org/10.1016/ $\underline{\mathrm{S} 0006-3207(03) 00096-\mathrm{X}}$

Verheyen K, Guntenspergen GR, Biesbrouck B, Hermy M. 2003. An integrated analysis of the effects of past land use on forest herb colonization at the landscape scale. Journal of Ecology 91: 731-742. DOI: https://doi.org/ 10.1046/j.1365-2745.2003.00807.x

Vellend M, Lechowicz MJ, Waterway MJ. 2000. Germination and establishment of forest sedges (Carex, Cyperaceae): tests for home-site advantage and effects of leaf litter. American Journal of Botany 87: 1517-1525. DOI: https://doi.org/10.2307/2656878

Wardle DA, Bonner KI, Barker GM. 2002. Linkages between plant litter decomposition, litter quality, and vegetation responses to herbivores. Functional Ecology 16: 585-595. DOI: https://doi.org/10.1046/j.1365-2435. 2002.00659.x

Zepeda-Gómez C, Lot A, Antonio-Némiga X, Manjarrez J. 2015. Evaluación del banco de semillas y su importancia en la rehabilitación de la vegetación de humedales del centro México. Botanical Sciences 93: 695-707. DOI: https://doi.org/10.17129/botsci.245

Zhu JJ, Matsuzaki T, Lee FG, Gonda Y. 2003. Effect of gap size created by thinning on seedling emergency, survival and establishment in a coastal pine forest. Forest Ecology and Management 182: 339-354. DOI: https:// doi.org/10.1016/S0378-1127(03)00094-X 